\title{
Progesterone receptor by immunohistochemistry and clinical outcome in breast cancer: a validation study
}

Syed K Mohsin ${ }^{1,2}$, Heidi Weiss ${ }^{1,2}$, Thomas Havighurst ${ }^{3}$, Gary M Clark ${ }^{1,2}$, Melora Berardo ${ }^{4}$, Le D Roanh ${ }^{5}$, Ta V To ${ }^{5}$, Qian Zho ${ }^{6}$, Richard R Love ${ }^{3}$ and D Craig Allred ${ }^{1,2}$

${ }^{1}$ The Breast Center, Baylor College of Medicine, Houston, TX, USA $;{ }^{2}$ Department of Pathology, The Methodist Hospital, Houston, TX, USA; ${ }^{3}$ Breast Cancer Program, University of Wisconsin, Madison, WI, USA; ${ }^{4}$ cKenna Memorial Hospital, New Braunfels, TX, USA; ${ }^{5}$ Hospital K and National Cancer Institute, Ha Noi, Vietnam and ${ }^{6}$ People's Hospital at Haimen City, Haimen City, China

Progesterone receptor is a surrogate marker of estrogen receptor activity in breast cancer and its utility in helping predict clinical outcome has been established using biochemical assays. However, most laboratories worldwide have switched to immunohistochemistry to assess progesterone receptor, but unfortunately no validated immunohistochemical assay exists for progesterone receptor. The purpose of this study was to develop and validate an immunohistochemical assay for progesterone receptor in breast cancer. The assay was based on monoclonal antibody 1294 (DakoCytomation) and slides were scored microscopically using the 'Allred score' on a scale of $0-8$. The assay was compared to ligand-binding assay in 1235 breast cancers, and a subset $(n=362)$ that received only hormonal therapy was used to define a cutoff for progesterone receptorpositive. Clinical utility was validated in an independent set of samples $(n=423)$ from a clinical trial randomizing premenopausal breast cancer patients to tamoxifen + oophorectomy vs observation following surgery. A cutoff of $>2$ (corresponding to $>1 \%$ positive cells) dichotomized patients with significantly better or worse clinical outcome $(P=\mathbf{0 . 0 0 1 4})$. Progesterone receptor by immunohistochemistry provided significantly better results than progesterone receptor by ligand-binding assay in predicting clinical outcome. In the clinical trial, a positive result in univariate analyses was associated with significantly improved disease-free and overall survival both in untreated (hazard ratios $/ P=0.656 / 0.060$ and $0.479 / 0.005$, respectively) and hormonally treated patients (hazard ratios $/ P=0.529 / 0.017$ and $0.451 / 0.007$, respectively). Positive progesterone receptor remained significant for improved disease-free and overall survival (hazard ratios $/ P=0.666 / 0.038$ and $0.549 / 0.007$, respectively) in multivariate analyses including the standard variables of tumor size, nodal status, treatment, histological grade, and HER-2/neu status. Estrogen and progesterone receptors are codependent variables and progesterone receptor was a weaker predictor of response to endocrine therapy than estrogen receptor when both were included in multivariate analysis. This is the first comprehensive study assessing the clinical usefulness of progesterone receptor by immunohistochemistry in archival tissue in breast cancer. Progesterone receptor assessed by immunohistochemistry provides useful information about clinical outcome and it is better than progesterone receptor measured by ligand-binding assay.

Modern Pathology (2004) 17, 1545-1554, advance online publication, 23 July 2004; doi:10.1038/modpathol.3800229

Keywords: breast; cancer; progesterone receptor; predictive factor; immunohistochemistry; validation study

Assessment of hormone receptors to predict clinical outcome in breast cancer has been an accepted standard for nearly two decades. The role of estrogen receptor (ER) as a prognostic and predictive

Correspondence: Dr SK Mohsin, MD, The Breast Center, Baylor College of Medicine, One Baylor Plaza, BCM-600, Houston, TX 77030, USA.

E-mail: smohsin@breastcenter.tmc.edu

Received 31 March 2004; revised 26 May 2004; accepted 27 May 2004; published online 23 July 2004 factor has been well established. The term prognostic factor is used to define any measurement available at the time of diagnosis or surgery that is associated with clinical outcome in the absence of systemic adjuvant therapy. On the other hand, the term predictive factor defines any measurement associated with response or lack of response to a particular therapy. The primary reason to assess ER in breast cancer is as a predictive factor for response to endocrine therapy. However, not all patients with ER-positive breast cancers derive benefit from this 
form of therapy. Therefore, additional markers for response to endocrine therapy have been sought. Since progesterone receptor (PR) expression is induced by ER, it has been studied as a surrogate marker for ER activity and has been used as an additional predictive factor for hormonal therapy in breast cancer. The results of overview analyses of randomized clinical trials in early breast cancer have shown that PR may add to the power of ER for predicting response to endocrine therapy. ${ }^{1} \mathrm{PR}$ also predicts response to endocrine therapy in metastatic breast cancer. ${ }^{2}$ Like ER, PR has been measured in breast cancer patients as a 'standard' biomarker for a long time, initially by ligand-binding assay and for over a decade by immunohistochemistry. Panels of experts for both the College of American Pathologists and the American Society of Clinical Oncology have recommended that both ER and PR must be measured in all primary breast cancers to select patients for therapeutic and adjuvant hormonal treatment. ${ }^{3,4}$ All of the studies reviewed by these panels to support these recommendations assessed PR by biochemical ligand-binding assay, which had been the gold standard to measure this biomarker in routine clinical practice.

However, most laboratories in the United States and throughout the world have switched to assessing ER and PR by immunohistochemistry on archival tissue since the mid 1990s, and no longer perform ligand-binding assay due to the potential advantages of immunohistochemistry (eg relatively low cost, time efficiency, and the ability to measure proteins directly on tumor cells). ${ }^{5}$ Unfortunately, most laboratories are using immunohistochemistry assays that do not meet the guidelines for technical and clinical validation, which are strongly recommended for routine clinical practice. ${ }^{6-8}$ Technical validation means that the assay used to measure a factor is sensitive, specific, reproducible, and interpreted in a relatively uniform manner between laboratories. Clinical validation means that the factor has been shown in studies, ideally randomized clinical trial, to identify subsets of patients with significantly different clinical outcome or response to treatment. A useful factor is one that is validated and used by physicians in daily practice to make treatment decisions. Panel of experts at College of American Pathologists, American Society of Clinical Oncology, and National Institute of Health in their last consensus statements had several reservations about assessing ER and PR by immunohistochemistry and noted a lack of standardized and validated assays. ${ }^{3,4,9}$

Although a few validated immunohistochemistry methodologies have been published for ER, ${ }^{10-14}$ none exist for PR. The few published studies that have assessed PR by immunohistochemistry consisted of patients of mixed clinical stages and variable treatment, making it impossible to separate the prognostic effects from the predictive value of PR. In addition, these studies used many different antibodies of varying sensitivity and specificity, a variety of other reagents, different scoring systems, and often arbitrary definitions of PR cut-offs to define positivity, making it extremely difficult to compare results.

The purpose of the present study was to develop and validate an immunohistochemical assay for PR in breast cancer, as we did previously for ER. ${ }^{13}$ We previously validated an immunohistochemical assay for PR on frozen sections using antibody KD $68 .{ }^{15}$ However, frozen section immunohistochemistry is not relevant today and the KD 68 antibody is no longer commercially available, prompting us to repeat the study on formalin-fixed, archival tissue sections with an available antibody. In this study, we developed an assay for PR by immunohistochemistry in archival breast cancer samples using antibody 1294, compared it to PR assessed by ligand-binding assay in the same samples, and determined its prognostic and predictive abilities in a clinical trial.

\section{Materials and methods}

\section{Patient Characteristics}

This study was conducted with approval of local Institutional Review Boards and accomplished in two phases following published guidelines for validation of prognostic and predictive factors. ${ }^{6-8}$ The first set of samples was designated as the 'test set'. This set of samples served to define the clinical utility of immunohistochemical assay, and establish the cutoff to define PR-positive and PR-negative in the subset of breast cancer patients who received endocrine therapy only. The 'test set' consisted of 1235 cases of primary breast cancers in which PR measurements by ligand-binding assay were available, thus allowing comparison of the two methods to assess PR. The patient characteristics of this cohort, which is a subset of our large tumor bank used in our previous studies of $\mathrm{ER}^{13}$ and PR on frozen sections, ${ }^{15}$ have been described in detail in earlier studies. Their characteristics are summarized in Table 1.

The second set, designated the 'validation set', was used to confirm the clinical utility of the immunohistochemical PR assay. It consisted of 423 premenopausal Vietnamese and Chinese women with stage II and III operable breast cancer treated with mastectomy (Table 1). These patients were a subset of 709 patients who participated in a randomized clinical trial of bilateral oophorectomy plus adjuvant tamoxifen for 5 years vs no adjuvant treatment, and whose clinical characteristics and location of participating centers were detailed in our previous report. ${ }^{14}$ None of these patients received any adjuvant cytotoxic chemotherapy. Archival paraffin blocks were available from $468(66 \%)$ of 709 patients. Prior to performing the immunohisto- 
Table 1 Characteristics of patients and breast cancers in test and validation sets

\begin{tabular}{|c|c|c|c|c|c|c|}
\hline & \multicolumn{3}{|c|}{ Test set } & \multicolumn{3}{|c|}{ Validation set } \\
\hline & $\begin{array}{c}\text { Overall } \\
\mathrm{n}=1235\end{array}$ & $\begin{array}{c}\text { Untreated } \\
\mathrm{n}=593\end{array}$ & $\begin{array}{c}\text { Endo only } \\
\mathrm{n}=362\end{array}$ & $\begin{array}{l}\text { Overall } \\
\mathrm{n}=423\end{array}$ & $\begin{array}{c}\text { Untreated } \\
\mathrm{n}=210\end{array}$ & $\begin{array}{c}\text { Endo only } \\
\mathrm{n}=213\end{array}$ \\
\hline \multicolumn{7}{|l|}{ Lymph nodes } \\
\hline Negative & $781(63 \%)$ & $495(83 \%)$ & $200(55 \%)$ & $175(43 \%)$ & $97(47 \%)$ & $78(38 \%)$ \\
\hline Positive & $454(37 \%)$ & $98(17 \%)$ & $162(45 \%)$ & $235(57 \%)$ & $109(53 \%)$ & $126(62 \%)$ \\
\hline Unknown & & & & 13 & 4 & 9 \\
\hline \multicolumn{7}{|l|}{ Tumor size $(\mathrm{cm})$} \\
\hline$\leq 2$ & $411(34 \%)$ & $221(38 \%)$ & $133(37 \%)$ & $68(16 \%)$ & $28(13 \%)$ & $40(19 \%)$ \\
\hline$>2-5$ & $630(51 \%)$ & $295(50 \%)$ & $183(51 \%)$ & $320(76 \%)$ & $166(79 \%)$ & $154(73 \%)$ \\
\hline$>5$ & $183(15 \%)$ & $68(12 \%)$ & $44(12 \%)$ & $33(8 \%)$ & $16(8 \%)$ & $17(8 \%)$ \\
\hline Unknown & 11 & & & 2 & 0 & 2 \\
\hline \multicolumn{7}{|l|}{ Histological type } \\
\hline Ductal (NOS) & $1057(86 \%)$ & $527(89 \%)$ & $301(83 \%)$ & $337(82 \%)$ & $164(80 \%)$ & $173(84 \%)$ \\
\hline Special types & $151(12 \%)$ & $46(8 \%)$ & $57(16 \%)$ & $66(16 \%)$ & $39(18 \%)$ & $27(13 \%)$ \\
\hline Others & $27(2 \%)$ & $20(3 \%)$ & $4(1 \%)$ & $10(2 \%)$ & $3(2 \%)$ & $7(3 \%)$ \\
\hline Unknown & & & & & 4 & 6 \\
\hline \multicolumn{7}{|l|}{ Age (years) } \\
\hline$<50$ & $334(27 \%)$ & $139(23 \%)$ & $39(11 \%)$ & $409(97 \%)$ & $203(97 \%)$ & $206(98 \%)$ \\
\hline$\geq 50$ & $901(73 \%)$ & $454(77 \%)$ & $323(89 \%)$ & $12(3 \%)$ & $7(3 \%)$ & $3(2 \%)$ \\
\hline Unknown & & & & 2 & 0 & 2 \\
\hline \multicolumn{7}{|c|}{ ER (immunohistochemistry) } \\
\hline Negative & $393(32 \%)$ & $192(33 \%)$ & $43(12 \%)$ & $164(39 \%)$ & $69(33 \%)$ & $95(45 \%)$ \\
\hline Positive & $820(68 \%)$ & $388(67 \%)$ & $313(88 \%)$ & $258(61 \%)$ & $140(67 \%)$ & $118(55 \%)$ \\
\hline Unknown & 22 & & & 1 & 1 & \\
\hline \multicolumn{7}{|c|}{ Adjuvant therapy } \\
\hline None & $593(48 \%)$ & & & $210(50 \%)$ & & \\
\hline Endo only & $362(29 \%)$ & & & $213(50 \%)$ & & \\
\hline Chemo only & $173(14 \%)$ & & & 0 & & \\
\hline Endo+chemo & 107 (9\%) & & & 0 & & \\
\hline \multicolumn{7}{|l|}{ Clinical outcome } \\
\hline Died & $506(41 \%)$ & $250(42 \%)$ & $139(38 \%)$ & $105(25 \%)$ & $58(28 \%)$ & $47(22 \%)$ \\
\hline Recurred & $406(33 \%)$ & $193(32 \%)$ & $104(29 \%)$ & $130(31 \%)$ & $78(37 \%)$ & $52(24 \%)$ \\
\hline \multicolumn{7}{|c|}{ Median $F / U$ of pts still alive (months) } \\
\hline & 89 & 100 & 81 & 44 & 42 & 47 \\
\hline
\end{tabular}

chemistry assay, these blocks were evaluated for overall quality, blinded to therapy or outcome. After this quality control, 423 cases were selected for this study. The remaining 45 cases were classified as 'unsatisfactory', which was usually due to inadequate number of tumor cells and/or very poor fixation. Patient and tumor characteristics of these patients were compared to the entire trial population and no statistically significant differences were found (data not shown), suggesting that this selection process did not introduce significant biases. A total of 213 patients were randomized to the treatment arm and 210 to the control arm, and their characteristics are summarized in Table 1.

\section{Biochemical Assay for PR}

PR levels were measured by the standard dextrancoated charcoal method as previously described ${ }^{16}$ and validated using level $\geq 5 \mathrm{fmol} / \mathrm{mg}$ protein as PR-positive. ${ }^{17}$

\section{Immunohistochemical Assay for PR}

As the initial step, we compared several PR antibodies; KD 68 (Abbott Diagnostics, IL, USA), 1A6 (DakoCytomation, CA, USA), Ab-8 (Neomarkers, CA, USA), 636 (DakoCytomation, CA, USA), and 1294 (DakoCytomation, CA, USA), to determine how well they performed on paraffin sections, using a battery of antigen retrieval conditions and optimizing sensitivity by adjusting the concentration of the primary antibody in order to detect a wide range of PR expression (from negative to highly positive). We then compared these antibodies in a set of 233 breast cancer samples in which PR status was already known by ligand-binding assay. Antibody 1294 showed the best range for detecting PR 
expression and had highest concordance (82\%) with PR by ligand-binding assay, and was therefore selected for this study (data not shown). Recently, a comprehensive study on several PR antibodies by Press et $a l^{18}$ has also shown the superiority of 1294 antibody.

\section{Immunohistochemical Staining in the Test and Validation Sets}

For the test set, tissue sections were prepared from pulverized frozen tumor specimens left over from the ligand-binding assay as previously described, ${ }^{19}$ with minor modifications. Owing to the ultracold temperature used during pulverization, the tissue was fractured into histologically intact fragments ranging from approximately 0.1 to $1.0 \mathrm{~mm}$ in size. Individual samples consisted of $100 \mathrm{mg}$ pellets of this particulate tissue, which was fixed for $8 \mathrm{~h}$ in $10 \%$ neutral buffered formalin and routinely processed to paraffin blocks. These uniformly prepared tissue samples have been used to validate many prognostic and predictive factors in breast cancer including $\mathrm{ER}^{13}$ and others. ${ }^{20,21}$ For the validation set, samples were fixed in 10\% neutral buffered formalin for 6-24h in most cases, processed, and embedded in paraffin at different centers in Vietnam and China.

The immunohistochemistry assay was performed on $4 \mu \mathrm{m}$ sections cut from the blocks and floatmounted on plus-coated glass slides (Fisher Scientific, TX, USA). The essential steps of immunohistochemistry assay included antigen retrieval in 0.9 M Tris-HCl buffer ( $\mathrm{pH}$ 9.0) in a pressure cooker for $10 \mathrm{~min}$; blocking endogenous peroxidase with $3 \%$ hydrogen peroxide; blocking nonspecific protein binding with an avidin-biotin blocking kit (Vector, CA, USA); incubating with primary mouse monoclonal antibody 1294 (DakoCytomation, CA, USA) at a dilution of 1:1600 for $1 \mathrm{~h}$ at room temperature; linking with biotinylated rabbit antimouse secondary antibody (DakoCytomation, CA, USA) at a dilution of 1:200 for $30 \mathrm{~min}$; enzyme labeling with freshly prepared horseradish peroxidase-labeled streptavidin (DakoCytomation, CA, USA) at a dilution of 1:200 for $30 \mathrm{~min}$; developing chromogen $\mathrm{DAB}+$ solution (DakoCytomation, CA, USA) for $15 \mathrm{~min}$; enhancing the chromogen with $0.2 \%$ osmium tetroxide; and lightly counterstaining the sections with $0.05 \%$ methyl green (Fisher Scientific, TX, USA). Human endocervix was used as a positive control because of its easy availability and relatively stable reactivity. The negative control consisted of nonimmune mouse IgG substituted for primary antibody. Controls were run with each batch of slides, at an average of 40 slides per batch. The method produced a distinct nuclear signal in PR-positive tumor cells (Figure 1). Complete protocols and reagent list can be found on our laboratory website (http://www.breastcenter.tmc.edu/cores/path).

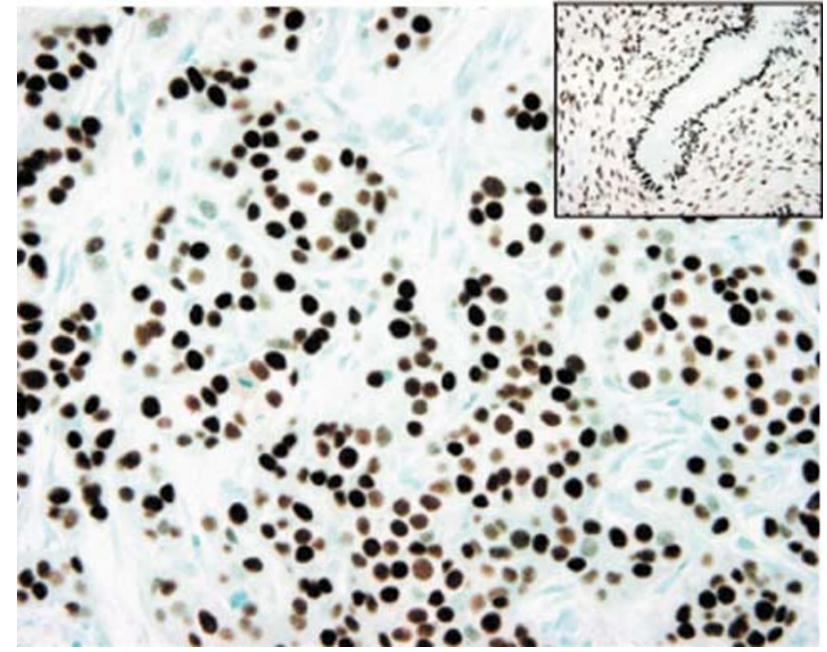

Figure 1 Progesterone receptor immunostaining on paraffinembedded invasive breast cancer, using antibody 1294. Inset: Nuclear staining in endocervical gland and stroma used as positive control $(\times 400)$.

\section{Interpretation of Immunostained Sections (Scoring System)}

Immunostained slides were evaluated by light microscopy and the immunohistochemistry signal was scored using the so-called 'Allred Score', as in our previous study assessing ER by immunohistochemistry $^{13}$ as well as several studies of other biomarkers. ${ }^{12,20,22-25}$ Briefly, a proportion score was assigned representing the estimated proportion of positive staining tumor cells $(0=$ none; $1<1 / 100$; $2=1 / 100$ to $<1 / 10 ; 3=1 / 10$ to $<1 / 3 ; 4=1 / 3-2 / 3$; $5=>2 / 3$ ). Average estimated intensity of staining in positive cells was assigned an intensity score ( 0 =none; 1 = weak; 2 =intermediate; $3=$ strong). Proportion score and intensity score were added to obtain a total score that ranged from $0-8$. This system is easy to learn and highly reproducible. ${ }^{13}$ Slides were scored by one pathologist (SKM) who did not have knowledge of ligand-binding assay results or patient outcome.

\section{Statistical Methods}

Distributions of categorical variables were compared using standard $\chi^{2}$ tests. Time to event or censor date was calculated from the date of diagnosis for the test set and date of study entry for the validation set. The definition of disease-free differs slightly between test set and validation set. For the test set, diseasefree is defined as first occurrence of recurrence or metastasis. Deaths without recurrence were not counted as events in the test set, as described previously. ${ }^{13}$ On the other hand, disease-free in validation set was defined as first occurrence of recurrence or death, if before recurrence. ${ }^{14}$ For overall survival, an event was defined as death from 
any cause. Patients with no event were censored at the last follow-up date.

An optimal cut point for PR positivity based on total immunohistochemistry score was obtained by using the minimum $P$-value approach. ${ }^{26}$ Since there are seven possible cut points from the immunohistochemistry total score (range: 0, 2-8), a Bonferroni procedure was employed which multiplies the $P$-values by seven in order to arrive at an adjusted $P$-value. ${ }^{27}$ The corresponding hazard ratios were also adjusted following the methods described before (27). Univariate and multivariate analyses for disease-free and overall survival used the logrank test and the Cox proportional hazards model and estimates of hazard ratios and $P$-values were adjusted for multiple significance as described previously. ${ }^{13}$ There has been strong evidence of a nonproportional effect of ER as indicated by the loss of its predictive value over long periods of patient follow-up. ${ }^{28}$ For this study, tests of proportionality of PR on disease-free and overall survival over the follow-up period were performed using hypothesis tests of PR as a time-dependent variable in the Cox model, as well as examining plots of the beta coefficients of PR from the Cox model vs follow-up time. ${ }^{29}$ For this study population, evidence of nonproportionality of PR was not detected, and Cox models were therefore developed without adjustment for $\mathrm{PR}$ as a time-dependent variable. The validation set was analyzed in a similar manner, without incorporating $P$-value or hazard estimate adjustments. Although the validation set was not powered to detect an interaction effect, an interaction term between treatment group and PR positivity status was included in the Cox model in order to evaluate prognostic and/or predictive effects of PR in this randomized trial. All statistical tests were two-sided at the 5\% level of significance, and were performed using the SAS Version 8.0.

\section{Results}

\section{Distribution of Immunohistochemistry Scores} and Concordance with Ligand-Binding Assay in the Test Set

In the test set, 525 tumors (43\%) showed no staining for PR (ie total score $=0$ ). Only a few cases showed staining at extreme ends of positive staining (ie $1.3 \%$ had a total score $=2$ and $2.6 \%$ had a total score $=8$ ). The rest of the immunohistochemistry scores were approximately uniformly distributed, ranging from 9 to $12 \%$ (Figure 2 ). We then did a head-to-head comparison of the immunohistochemistry and ligand-binding assay for concordance of results in 1219 cases in which PR ligand-binding assay was available. There was $86 \%$ concordance between the two methods: 583 cases (48\%) were positive and 470 cases (38\%) were negative by both

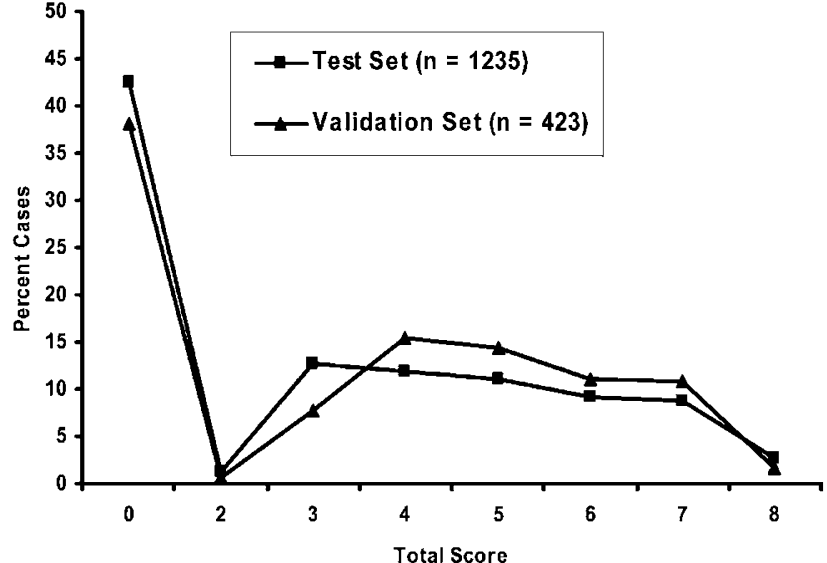

Figure 2 Distribution of PR immunohistochemical score in the samples.

Table 2 Comparison of PR status between ligand-binding assay and immunohistochemistry in the test set

\begin{tabular}{lcc}
\hline & \multicolumn{2}{c}{ Immunohistochemistry } \\
\cline { 2 - 3 } & Positive & Negative \\
\hline Ligand-binding assay & & \\
Positive & $583(48 \%)$ & $71(6 \%)$ \\
Negative & $95(8 \%)$ & $470(38 \%)$ \\
\hline
\end{tabular}

Kappa statistic $=0.72 .95 \%$ CI $(0.69,0.76)$.

methods. Among the 14\% discordant cases, 8\% were positive by immunohistochemistry only and $6 \%$ by ligand-binding assay only (Table 2). There was thus good agreement between the two methods, yielding a kappa statistic of 0.72 .

\section{Determination of Cutoff Value to Define PR-Positive}

The cutoff to segregate patients into clinically PRpositive vs clinically PR-negative was determined by univariate cutpoint analysis using both diseasefree and overall survival as the end points in the test set. The analysis was performed in two groups of patients: those receiving no adjuvant therapy and those who received endocrine treatment only (Figure 3). Preliminary analyses of the association of PR immunohistochemistry using proportion score and intensity score on diseasefree and overall survival in a Cox regression model indicated that neither proportion score nor intensity score alone were strongly associated with patient outcome. Therefore, cutpoint analysis was only performed for PR immunohistochemistry total score. The value of the total score that yielded the smallest log-rank $P$-value was chosen as the optimal cut point to define PR positivity. PR was a weak prognostic factor (overall survival only) for patients who received no adjuvant 
therapy. The best cutoffs and corresponding $P$ values for disease-free and overall survival were total score $=4 \quad(P=0.053$, adjusted $P=0.371)$ and total $\quad$ score $=5 \quad(P=0.003$, adjusted $P=0.021)$, respectively. Among patients who received adjuvant endocrine therapy, however, PR was a strong predictor of both disease-free and overall survival. The best cutoff for both end points was total score $>2$, with $P$-values 0.0003 (adjusted $P=$ 0.0021 ) and 0.0002 (adjusted $P=0.0014$ ), respectively (Figure 3). Therefore, in all subsequent analyses, we classified tumors as PR-positive if the total score by immunohistochemistry was greater than 2 and PR-negative if the total score was 0 or 2 . Of interest, this was the same cutoff value established in our previous study of ER by immunohistochemistry. ${ }^{13}$

\section{Association of PR by Immunohistochemistry with Other Clinical and Pathological Variables}

In the test set, PR positivity was significantly associated with node-negative disease $(P=0.008)$, special histological tumor types $(P=0.0042)$, smaller tumor size, postmenopausal status (age $>50$ years), diploid tumors, low S-phase, ER by ligand-

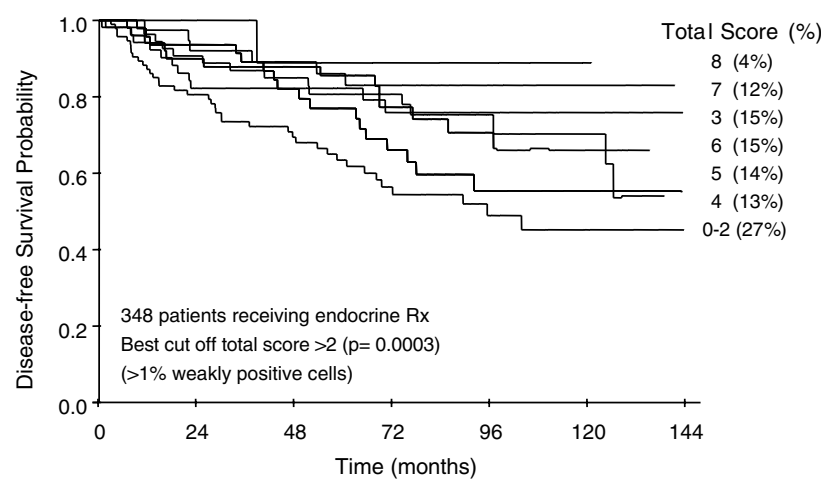

Figure 3 Determination of cutoff value to define PR-positive. These are Kaplan-Meier curves for disease-free survival $(n=348)$ corresponding to each possible total score by immunohistochemistry. Note the separation between patients with total score of 0 and 2 vs $\geq 3$. Tumors with a total score of $>2$ were defined as PR-positive. binding assay and immunohistochemistry, PR by ligand-binding assay as well as endocrine and chemotherapy (all $P$-values $<0.0001$ ). Association between PR as assessed by immunohistochemistry and other clinical and pathological variables in the validation set are displayed in Table 3.

\section{Comparison of Prognostic and Predictive Abilities of PR by Immunohistochemistry and Ligand-Binding Assay in the Test Set}

Multivariate Cox regression models including nodal status, tumor size, and age were used to compare the abilities of PR by immunohistochemistry and ligand-binding assay to predict clinical outcome. The results are summarized in Table 4, showing that PR measured either by immunohistochemistry or ligand-binding assay was not a significant prognostic factor in untreated patients. However, PR was a strong predictive factor as seen in the subset of patients receiving endocrine therapy only $(n=362)$ and those receiving both endocrine and cytotoxic chemotherapy $(n=107)$. PR positivity by immunohistochemistry was an independent predictor of improved disease-free $(\mathrm{HR}=0.546, P=0.0034)$ and overall survival $(\mathrm{HR}=0.595, P=0.0040)$ in endocrine-treated patients. Since the cutpoint for PR positivity by immunohistochemistry was determined from the same patient subset, adjusted HRs and $P$-values are also presented in Table 3. Similar analyses of PR assessed by ligand-binding assay in the same patients showed marginally significant ability to predict disease-free $(\mathrm{HR}=0.673$, $P=0.0534)$ and a significant ability to predict overall survival $(\mathrm{HR}=0.641, P=0.0124)$. In both settings, the predictive ability of PR by immunohistochemistry was stronger than PR by ligand-binding assay, both in the group of patients who received endocrine therapy only as well as those who got both endocrine and chemotherapy (Table 4). In a multivariate analysis including both PR by immunohistochemistry and PR by ligand-binding assay, along with tumor size, nodal status, and age, PR by immunohistochemistry remained significantly associated with disease-free $(\mathrm{HR}=0.539, P=0.0029)$ and overall survival ( $\mathrm{HR}=0.616, P=0.0078)$, while

Table 3 Association between PR and other clinical and pathological variables in the validation set

\begin{tabular}{lcccc}
\hline & No. of patients & PR-negative (mean or \%) & PR-positive (mean or \%) & $\chi^{2}$ (P-value) \\
\hline Tumor size & 466 & 3.59 & 3.23 & 0.0027 \\
Nodal status & 455 & 54 & 56.45 & NS \\
Age & 466 & 41.07 & 41.43 & NS \\
Histological grade & 351 & 13.57 & 9.83 & NS \\
ER-positive & 467 & 20 & 56 & 0.001 \\
Menstrual status & 366 & 58 & 26.48 & NS \\
HER2/neu & 459 & 50.0 & 53 & 0.001 \\
\hline
\end{tabular}

${ }^{\mathrm{a}}$ Tumor size, nodal status, and age were analyzed as continuous variables and the numbers represent means in each group. The other factors were analyzed as categorical variables and numbers represent the percentage in each group. 
Table 4 Multivariate cox regression models to compare prognostic and predictive ability of PR results by ligand-binding assay and immunohistochemistry in the test set

\begin{tabular}{|c|c|c|c|c|c|c|}
\hline & \multirow[b]{2}{*}{$N$} & & \multicolumn{2}{|c|}{ Disease-free survival } & \multicolumn{2}{|c|}{ Overall survival } \\
\hline & & & HR (adjusted HR) & $\mathrm{P}$-value (adjusted $\mathrm{P}$-value) & HR (adjusted HR) & $\mathrm{P}$-value (adjusted $\mathrm{P}$-value) \\
\hline \multirow[t]{2}{*}{ Untreated $^{\mathrm{a}}$} & 593 & IHC & 0.861 & 0.3110 & 0.835 & 0.1682 \\
\hline & & LBA & 0.825 & 0.1968 & 0.944 & 0.6657 \\
\hline \multirow[t]{2}{*}{ Endocrine therapy only ${ }^{\mathrm{b}}$} & 362 & IHC & $0.546(0.626)$ & $0.0034(0.0238)$ & $0.595(0.673)$ & $0.0040(0.0280)$ \\
\hline & & LBA & 0.673 & 0.0534 & 0.641 & 0.0124 \\
\hline \multirow[t]{2}{*}{ Endo+chemotherapy ${ }^{\mathrm{c}}$} & 107 & IHC & $0.309(0.392)$ & $0.0007(0.0049)$ & $0.417(0.515)$ & $0.0043(0.0301)$ \\
\hline & & LBA & 0.481 & 0.0291 & 0.557 & 0.0538 \\
\hline
\end{tabular}

IHC = immunohistochemistry; LBA = ligand-binding assay.

${ }^{\mathrm{a}}$ Includes nodal status as a significant factor in the disease-free model and nodal status, tumor size and age as significant factors in the overall survival model. Adjusted hazard ratio and $P$-value not calculated since PR was not significant in the model.

${ }^{\mathrm{b}}$ Includes nodal status, tumor size and age as significant factors in the disease-free model and nodal status and tumor size as significant factors in the overall survival model.

${ }^{\mathrm{C}}$ Includes nodal status as significant factors in the model.

PR by ligand-binding assay did not remain significant in the model.

\section{Prognostic and Predictive Ability of PR in the Validation Set}

The study design in the validation set provided us the opportunity to confirm the reproducibility, usefulness, and applicability of this assay to an independent set of patients. Using Kaplan-Meier curves, in the control group, patients with PR-positive tumors showed 9 and $16 \%$ improvements in disease-free and overall survival at 5 years, with $P$-values of 0.058 and 0.004 , respectively. In the treatment arm, patients with PR-positive tumors showed 9 and $13 \%$ improvement in disease-free and overall survival at 5 years due to endocrine therapy with $P$-values of 0.015 and 0.005 , respectively (Figure 4).

In Cox multivariate models of the entire patient population, containing nodal status, treatment, tumor size, histological grade, and Her-2/neu status, PR by immunohistochemistry remained an independent predictor of clinical outcome with $P$-values of 0.038 (hazard ratio 0.67) and 0.007 (hazard ratio 0.55) for disease-free and overall survival, respectively (Table 5). In the model that also included ER as a variable, PR did not remain significant, perhaps due to either imbalance in ER distribution in the two arms or ER and PR being highly correlated and thus not contributing independently (data not shown). A test of interaction between PR status and treatment was included in the overall model to determine if PR provides additional prognostic information with hormonal treatment. The hazard ratio for interaction term translated to an interaction hazard ratio equal to $1.13 .^{30}$ For $80 \%$ power and $5 \%$ significance level, the available sample size in the validation set could only detect an interaction hazard ratio of at least 2.8, as opposed to the observed 1.13 in our multivariate model, ${ }^{30}$ suggesting that this analysis was underpowered to show an independent predictive effect of PR.

\section{Discussion}

ER and PR status as measured by biochemical ligand-binding assay are the only prognostic and predictive biomarkers recommended for routine clinical use in breast cancer by the American Society of Clinical Oncology and the College of American Pathologists. ${ }^{3,4}$ Both are relatively weak prognostic factors, but strong predictive factors for response to adjuvant and therapeutic hormonal therapy. The primary reason for measuring these biomarkers today is the latter. These recommendations, including the overview analysis of randomized clinical trials in early breast cancer, ${ }^{1}$ are based on standardized ligand-binding assay methodology. However, ligand-binding assay has been replaced by immunohistochemistry on archival tissue over the past several years. Several recent studies have shown that assessing ER by immunohistochemistry is as good as or better than the ligand-binding assay, ${ }^{10-13}$ but similar validation studies for PR are lacking.

There are several reasons that comprehensive studies of PR by immunohistochemistry lagged behind that for ER. First, many clinicians depend on ER status alone to select patients for hormonal therapy. PR alone was found to be a weaker prognostic and predictive factor as compared to ER in studies using ligand-binding assay. ${ }^{1}$ In addition, until relatively recently, there were only a limited number of good antibodies available for PR that worked on archival tissue. Despite these limitations, there have been several studies of assessing PR by immunohistochemistry in various settings in breast cancer. For example, three studies ${ }^{15,31,32}$ assessed 
Control arm
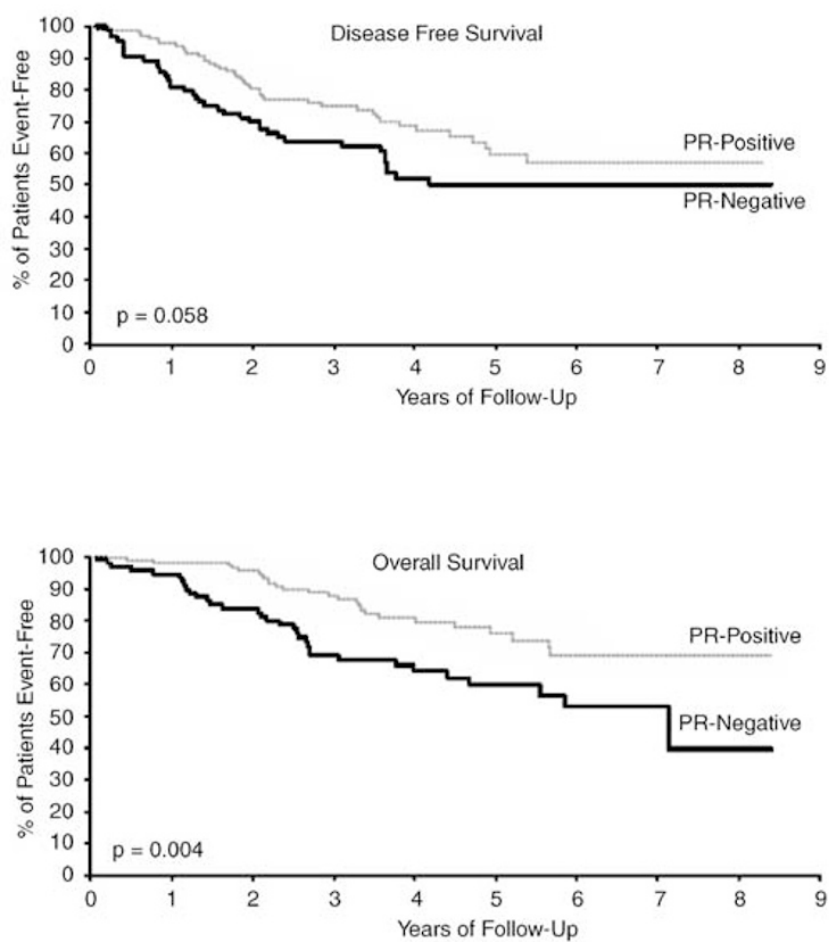

Treated arm
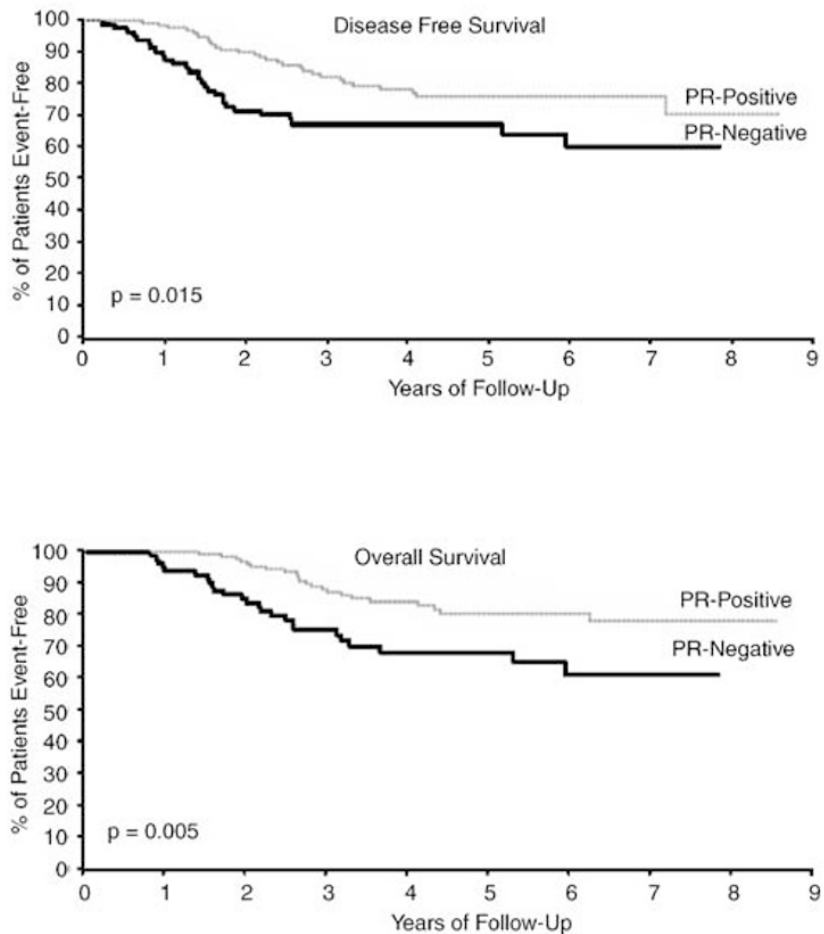

Figure 4 Clinical outcome and PR in the validation set: Kaplan-Meier survival curves for disease-free and overall survival. In the control arm $(n=210)$, there was 9 and $16 \%$ survival advantage at 5 years for disease-free and overall survival, respectively, as compared to 9 and $13 \%$ benefit in the treatment arm $(n=213)$.

Table 5 Multivariate cox regression analysis in the validation set

\begin{tabular}{|c|c|c|c|c|}
\hline & \multicolumn{2}{|c|}{$\begin{array}{l}\text { Disease-free survival } \\
\text { Events }=113^{\mathrm{a}}\end{array}$} & \multicolumn{2}{|c|}{$\begin{array}{l}\text { Overall survival } \\
\text { Events }=89^{\mathrm{a}}\end{array}$} \\
\hline & $H R$ & P-value & $H R$ & $\mathrm{P}$-value \\
\hline $\mathrm{PR}$ & 0.666 & 0.0384 & 0.549 & 0.0067 \\
\hline Treatment & 0.621 & 0.0131 & 0.737 & 0.1599 \\
\hline Nodal status & 1.355 & 0.0003 & 1.372 & 0.0014 \\
\hline Tumor size & 1.143 & 0.0216 & 1.133 & 0.0535 \\
\hline Histological grade & 1.779 & 0.0201 & 1.764 & 0.0423 \\
\hline HER-2/neu & 1.147 & 0.4861 & 1.514 & 0.0597 \\
\hline
\end{tabular}

${ }^{\mathrm{a}}$ Total number of patients in this analysis is 363 .

patients receiving adjuvant hormonal therapy alone, and all three showed a significant relationship between PR positivity and improved outcome. However, two were based on frozen section immunohistochemistry ${ }^{15,31}$ and the other ${ }^{32}$ used the KD 68 antibody, rendering them irrelevant today because laboratories exclusively use fixed archival tissue and because the KD 68 antibody is no longer commercially available. A total of 12 studies looked at patients receiving combined hormonal and cytotoxic chemotherapy. ${ }^{33-44}$ Only seven ${ }^{33-35,39,40,42,44}$ showed a significant relationship between PR status and clinical benefit. Of these positive studies, four ${ }^{34,35,39,40}$ used frozen section immunohistochemistry, and the other three, which were done on archival tissue, used either KD $68^{42}$ or noncommercial antibodies. ${ }^{33,44}$ Similarly, four studies, ${ }^{12,31,35,45}$ evaluated patients with advanced/metastatic disease, and three ${ }^{12,31,35}$ showed a significant clinical benefit of hormonal therapy in patients with PRpositive tumors. All three studies employed the KD 68 antibody. Looking at these studies as a group, over $60 \%$ were based on frozen section immunohistochemistry, and about the same number on the KD 68 antibody. Collectively, these studies were based on five different antibodies, and used six different 
methods of scoring with arbitrary cutoffs to define PR positivity, ranging from 1 to $50 \%$ ! Clearly, this body of work falls far short of the guidelines for validation of prognostic and predictive factors. ${ }^{6-8}$

Our goal was to develop an accurate and reliable assay to assess PR by immunohistochemistry in archival tissue based on commercially available reagents at reasonable cost, and to validate the clinical usefulness of this assay compared to the gold standard ligand-binding assay. In our test set, we have demonstrated that PR by immunohistochemistry provides significantly better clinical information as compared to ligand binding assay. In addition, an important finding of this study is the low optimum cutoff to define PR-positive (total score $>2$, corresponding to $>1 \%$ weakly positive tumor cells). Of interest, in our previous validation of ER by immunohistochemistry in breast cancer, total score $>2$ was also determined to be the optimum cutpoint. ${ }^{13}$ In the present study, PR total score $=3$ (the lowest positive score, corresponding to $1-10 \%$ weakly positive tumor cells) comprised $15 \%$ of the endocrine-treated patients (Figure 3). Most laboratories arbitrarily use $10 \%$ as a cutoff to define PR-positive. Thus, our results suggest that using such a high arbitrary cutoff can lead to misclassification of as many as $15 \%$ of breast cancer patients as PR-negative. Finally, we have demonstrated the clinical usefulness of this assay in an independent set of patients in a randomized clinical trial. This validation set did not have enough statistical power for analyses of treatment interaction, or for evaluation of PR in relation to ER (ie value of $P R$ in ER-negative tumors). This latter aspect of PR and its utility in ER-negative cancers has been analyzed in detail in our two other large databases by Bardou et al, ${ }^{46}$ but PR in those samples was assessed by ligand-binding assay.

In summary, we have developed and validated an immunohistochemical assay for $\mathrm{PR}$ in archival tissue that is sufficiently validated to justify routine clinical use in the management of breast cancer patients. However, as compared to ER, PR adds only a limited amount of additional predictive information for response to hormonal therapy. We demonstrated that patients with even very low levels of PR (1-10\% weakly positive cells) have a better clinical outcome. This study suggests that PR by immunohistochemistry can provide useful clinical information that is better than ligand-binding assay. Laboratories performing assessment of PR in routine clinical practice should either comprehensively validate their assay or adopt a validated assay, in order to report reliable and clinically meaningful results for the clinicians.

\section{Acknowledgements}

This work was supported by Grants NIH P50-CA 58183 and NIH/NCI CA 64339.

\section{References}

1 Early Breast Cancer Trialists' Collaborative Group. Tamoxifen for early breast cancer: an overview of the randomised trials. Lancet 1998;351:1451-1467.

2 Ravdin PM, Green S, Dorr TM, et al. Prognostic significance of progesterone receptor levels in estrogen receptor-positive patients with metastatic breast cancer treated with tamoxifen: results of a prospective Southwest Oncology Group study. J Clin Oncol 1992;10: 1284-1291.

3 Fitzgibbons PL, Page DL, Weaver D, et al. Prognostic factors in breast cancer. College of American Pathologists Consensus Statement 1999. Arch Pathol Lab Med 2000;124:966-978.

4 American Society of Clinical Oncology. 1997 update of recommendations for the use of tumor markers in breast and colorectal cancer. Adopted on November 7 1997 by the American Society of Clinical Oncology J Clin Oncol 1998;16:793-795.

5 Allred DC. Should immunohistochemical examination replace biochemical hormone receptor assays in breast cancer? Am J Clin Pathol 1993;99:1-3.

6 McGuire WL. Breast cancer prognostic factors: evaluation guidelines. J Natl Cancer Inst 1991;83:154-155.

7 Gasparini G, Pozza F, Harris AL. Evaluating the potential usefulness of new prognostic and predictive indicators in node-negative breast cancer patients. J Natl Cancer Inst 1993;85:1206-1219.

8 Hayes DF, Bast RC, Desch CE, et al. Tumor marker utility grading system: a framework to evaluate clinical utility of tumor markers. J Natl Cancer Inst 1996;88: 1456-1466.

9 NIH. Adjuvant therapy for breast cancer. NIH consensus statement 2000;17:6-7.

10 Alberts SR, Ingle JN, Roche PR, et al. Comparison of estrogen receptor determinations by a biochemical ligand-binding assay and immunohistochemical staining with monoclonal antibody ER1D5 in females with lymph node positive breast carcinoma entered on two prospective clinical trials. Cancer 1996;78:764-772.

11 Clahsen PC, van de Velde CJ, Duval C, et al. The utility of mitotic index, oestrogen receptor and Ki-67 measurements in the creation of novel prognostic indices for node-negative breast cancer. Eur J Surg Oncol 1999;25:356-363.

12 Elledge RM, Green S, Pugh R, et al. Estrogen receptor (ER) and progesterone receptor (PgR), by ligandbinding assay compared with ER, PgR and pS2, by immuno-histochemistry in predicting response to tamoxifen in metastatic breast cancer: a Southwest Oncology Group Study. Int J Cancer 2000;89:111-117.

13 Harvey JM, Clark GM, Osborne CK, et al. Estrogen receptor status by immunohistochemistry is superior to the ligand-binding assay for predicting response to adjuvant endocrine therapy in breast cancer. J Clin Oncol 1999;17:1474-1481.

14 Love RR, Duc NB, Allred DC, et al. Oophorectomy and tamoxifen adjuvant therapy in premenopausal Vietnamese and Chinese women with operable breast cancer. J Clin Oncol 2002;20:2559-2566.

15 Berardo M, Clark GM, de Moor C, et al. Prognostic and predictive properties of immunohistochemical progesterone receptors in breast cancer. Proc Am Soc Clin Oncol 1995;14:110 (abstract \#139).

16 Chamness GC, McGuire WL. Methods for analyzing steroid receptors in human breast cancer. In: McGuire 
WL (ed). Breast Cancer. Advances in Research and Treatment. Plenum: New York: 1979, p 149.

17 Clark GM, Osborne CK, McGuire WL. Correlations between estrogen receptor, progesterone receptor, and patient characteristics in human breast cancer. J Clin Oncol 1984;2:1102-1109.

18 Press M, Spaulding B, Groshen S, et al. Comparison of different antibodies for detection of progesterone receptor in breast cancer. Steroids 2002;67:799-813.

19 Allred DC, Clark GM, Tandon AK, et al. Immunohistochemistry on histological sections made from small (50 mg) samples of pulverized breast cancer. J Histotechn 1993;16:117-120.

20 Allred DC, Clark GM, Elledge R, et al. Association of p53 protein expression with tumor cell proliferation rate and clinical outcome in node-negative breast cancer. J Natl Cancer Inst 1993;85:200-206.

21 Brown RW, Allred CD, Clark GM, et al. Prognostic value of Ki-67 compared to S-phase fraction in axillary node-negative breast cancer. Clin Cancer Res 1996;2:585-592.

22 Elledge RM, Clark GM, Fuqua SA, et al. p53 protein accumulation detected by five different antibodies: relationship to prognosis and heat shock protein 70 in breast cancer. Cancer Res 1994;54:3752-3757.

23 O’Malley FP, Saad Z, Kerkvliet N, et al. The predictive power of semiquantitative immunohistochemical assessment of p53 and c-erb B-2 in lymph node-negative breast cancer. Hum Pathol 1996;27:955-963.

24 Ellis MJ, Coop A, Singh B, et al. Letrozole is more effective neoadjuvant endocrine therapy than tamoxifen for ErbB-1- and/or ErbB-2-positive, estrogen receptor-positive primary breast cancer: evidence from a phase III randomized trial. J Clin Oncol 2001;19: 3808-3816.

25 Regitnig P, Reiner A, Dinges HP, et al. Quality assurance for detection of estrogen and progesterone receptors by immunohistochemistry in Austrian pathology laboratories. Virchows Arch 2002;441: 328-334.

26 Mazumdar M, Glassman JR. Categorizing a prognostic variable: review of methods, code for easy implementation and applications to decision-making about cancer treatments. Stat Med 2000;19:113-132.

27 Hilsenbeck SG, Clark GM. Practical $P$-value adjustment for optimally selected cutpoints. Stat Med 1996;15:103-112.

28 Pichon MF, Broet $\mathrm{P}$, Magdelenat $\mathrm{H}$, et al. Prognostic value of steroid receptors after long-term follow-up of 2257 operable breast cancers. Br J Cancer 1996;73: 1545-1551.

29 Therneau TM, Grambsch PM. Modeling survival data: Extending the Cox model. Springer-Verlag: New York: 2000.

30 Peterson B, George SL. Sample size requirements and length of study for testing interaction in a $2 \times k$ factorial design when time-to-failure is the outcome [corrected]. Control Clin Trials 1993;14:511-522.

31 Pertschuk LP, Feldman JG, Eisenberg KB, et al. Immunocytochemical detection of progesterone receptor in breast cancer with monoclonal antibody. Relation to biochemical assay, disease-free survival, and clinical endocrine response. Cancer 1988;62: 342-349.

32 Knoop AS, Bentzen SM, Nielsen MM, et al. Value of epidermal growth factor receptor, HER2, p53, and steroid receptors in predicting the efficacy of tamox- ifen in high-risk postmenopausal breast cancer patients. J Clin Oncol 2001;19:3376-3384.

33 Seymour L, Meyer K, Esser J, et al. Estimation of PR and ER by immunocytochemistry in breast cancer. Comparison with radioligand binding methods. Am J Clin Pathol 1990;94:S35-40.

34 Reiner A, Neumeister B, Spona J, et al. Immunocytochemical localization of estrogen and progesterone receptor and prognosis in human primary breast cancer. Cancer Res 1990;50:7057-7061.

35 Pertschuk LP, Kim DS, Nayer K, et al. Immunocytochemical estrogen and progestin receptor assays in breast cancer with monoclonal antibodies. Histopathologic, demographic, and biochemical correlations and relationship to endocrine response and survival. Cancer 1990;66:1663-1670.

36 Gasparini G, Pozza F, Dittadi R, et al. Progesterone receptor determined by immunocytochemical and biochemical methods in human breast cancer. J Cancer Res Clin Oncol 1992;118:557-563.

37 Hurlimann J, Gebhard S, Gomez F. Oestrogen receptor, progesterone receptor, pS2, ERD5, HSP27 and cathepsin $\mathrm{D}$ in invasive ductal breast carcinomas. Histopathology 1993;23:239-248.

38 Kommoss F, Pfisterer J, Idris T, et al. Steroid receptors in carcinoma of the breast. Results of immunocytochemical and biochemical determination and their effects on short-term prognosis. Anal Quant Cytol Histol 1994;16:203-210.

39 Beck T, Weikel W, Brumm C, et al. Immunohistochemical detection of hormone receptors in breast carcinomas (ER-ICA, PgR-ICA): prognostic usefulness and comparison with the biochemical radioactive-ligandbinding assay (DCC). Gynecol Oncol 1994;53:220-227.

40 Ferrer Roca OF, Ramos A, Diaz Cardama A. Immunohistochemical correlation of steroid receptors and disease-free interval in 206 consecutive cases of breast cancer: validation of telequantification based on global scene segmentation. Anal Cell Pathol 1995;9:151-163.

41 Stierer M, Rosen H, Weber $\mathrm{R}$, et al. A prospective analysis of immunohistochemically determined hormone receptors and nuclear features as predictors of early recurrence in primary breast cancer. Breast Cancer Res Treat 1995;36:11-21.

42 MacGrogan G, Bonichon F, de Mascarel I, et al. Prognostic value of p53 in breast invasive ductal carcinoma: an immunohistochemical study on 942 cases. Breast Cancer Res Treat 1995;36:71-81.

43 Layfield LJ, Saria EA, Conlon DH, et al. Estrogen and progesterone receptor status determined by the Ventana ES 320 automated immunohistochemical stainer and the CAS 200 image analyzer in 236 early-stage breast carcinomas: prognostic significance. J Surg Oncol 1996;61:177-184.

44 Gago FE, Tello OM, Diblasi AM, et al. Integration of estrogen and progesterone receptors with pathological and molecular prognostic factors in breast cancer patients. J Steroid Biochem Mol Biol 1998;67:431-437.

45 Muller-Holzner E, Zeimet AG, Daxenbichler G, et al. Progesterone receptors in routinely paraffin-embedded primary breast carcinomas and lymph node metastases. Breast Cancer Res Treat 1993;25:47-55.

46 Bardou VJ, Arpino G, Elledge RM, et al. Progesterone receptor status significantly improves outcome prediction over estrogen receptor status alone for adjuvant endocrine therapy in two large breast cancer databases. J Clin Oncol 2003;21:1973-1979. 